\title{
Differential Sensibility of Information Processing Capacity with Age: Effects of Physical Activity and Task Complexity ${ }^{*}$
}

\author{
Khadher Souha ${ }^{1}$, Abedelmalek Salma ${ }^{2 \#}$, Grira Youssef ${ }^{3}$, Bertsch Jean ${ }^{4}$ \\ ${ }^{1}$ Laboratory of Psychology of Physical Practice Complexity, Innovation, in Motor Activities \\ (CIAMS), University of Paris XI, Paris, France \\ ${ }^{2}$ Department of Physiology, Sousse Faculty of Medicine, Sousse, Tunisia \\ ${ }^{3}$ Research Unit, High Institute of Sport and Physical Education, Tunis, Tunisia \\ ${ }^{4}$ L’Université de Limoges, Limoges, France \\ Email: " s_abedelmalek@yahoo.fr
}

Received November 12, 2012; revised December 15, 2012; accepted December 23, 2012

\begin{abstract}
Study Aim: Movement control systems are altered by the aging process. Numerous researches have explained the changes that occur with aging, and many of those changes are related to central nervous system (CNS) effects. This article evaluates the impact of age, the practice of regular physical activity, and the task complexity on decision-making ability. Methods: 120 healthy male subjects volunteered to participate in this study. They included 60 young adults (i.e., 30 sedentary and 30 active) (age: $24.35 \pm 2.82$ years), as well as 60 older adults (age: $66.42 \pm 4.06$ years) (i.e., 30 sedentary and 30 active). They performed two types of tasks (i.e., simple and complex) to measure reaction time (RT). Subjects perceive visual stimuli through the computer screen. Results: Our results showed that older active subjects have lower RT than older sedentary subjects $(p<0.05)$. However, no significant difference was observed in young adults. Moreover, young adults had significantly lower RT than older subjects $(p<0.05)$. Besides, we observed a significant increase in the RT when task is complex compared to the simple task in all groups. In addition, active adults have better RT regardless the complexity of the task (i.e., simple or complex). Conclusions: Physical activity improves the decision making ability in older subjects.
\end{abstract}

Keywords: Aging; Physical Activity; Complexity; Decision Making

\section{Introduction}

Aging can be characterized by decrements in a variety of cognitive processes [1,2]. In this context, numerous studies related to anatomical and physiological degradation that occurs with aging have shown evidence of age-related reduction in cerebral cortical volume [1-4], and changes in white matter integrity in healthy older adults [5,6]. However, several studies observed that cognitive performance is relatively stable with aging [7-9], although some metrics of sensory performance (e.g., thresholds) degrade [10-13]. Dinse et al. [14] observed that restoration of function in the aging population is attainable due to the emergence of new processing strategies, and they attributed this to brain plasticity being operational in the aging population. Recently, Greenwood [15] has hypothesised that with aging, although there is significant evidence of both anatomical and

\footnotetext{
*Declaration of interest: The authors report no conflict of interest. The authors alone are responsible for the content and writing of the article. "Corresponding author.
}

physiological decline, there is no, or even negative, correlation with cognitive performance. Greenwood largely attributed the undefined compensatory mechanism that allows for maintenance of cortical information processing capacity to cortical plasticity $[15,16]$.

However, the impact of aging is not the same for all individuals, and some factors related to lifestyle could modulate its effects (i.e., age, sexe, physical activity or sedentarity). In this regard, physical activity is implicated in maintaining and enhancing multiple aspects of psychological functioning, and many transversal studies showed a positive relationship between physical activity and cognitive functions [17]. Furthermore, physical activity is the most important factor that improves the management of health and prevention of the negatives effects of aging [18]. Also, some authors concluded that physical practice is beneficial for improving cognitive abilities [19,20]. Conversely, Emery et al. [21] showed no significant effect of physical activity on cognitive abilities of the elderly. 
Moreover, the majority of studies that showed a beneficial impact of physical activity have separately investigated categories of cognitive functions such as inductive reasoning, attentional resources, reaction time, learning or psychomotor function [22-26]. Hence, the goal of this study was to examine the relationship between physical activity and performances on a large panel of neuropsychological tests assessing the main categories of cognitive processes within one experiment.

Different studies have revealed beneficial effects of a higher level of physical activity on some cognitive functions that are generally affected by aging. However, all the main cognitive domains have not been generally examined in the same study. It is critical therefore to investtigate the differential sensibility of information processing capacity with age. Thus, the aim of this study was to evaluate the impact of age, the practice of regular physical activity, and the task complexity on decision-making ability. We hypothesized that decision-making is better in older active than older sedentary adults and that older athletes are not differentially affected by task complexity.

\section{Methods}

\subsection{Participants}

120 healthy male subjects took part in this study after giving their written informed consent. They included 60 young adults (i.e., 30 sedentary and 30 active) (age: $24.35 \pm 2.82$ years), as well as 60 older adults (age: 66.42 \pm 4.06 years) (i.e., 30 sedentary and 30 active). The study was performed in accordance with Declaration of Helsinki and the experimental procedures were reviewed and approved in advance by an institutional review board. A survey about medication and medical history was filled out by each subject before experimental tests to exclude subjects with a history of neurological impairment. Subjects reported no sleep disorder, or any major health problems (i.e., history of neurological or psychiatric illness, cancer, and cardiovascular disease), normal, or corrected-to-normal vision and hearing, and none of them was taking any medication. Characteristics of the sub- jects are shown in Table 1.

\subsection{Evaluation of Physical Activity}

Health, educational level and amount of physical activity were evaluated on the basis of the answers to two valid questionnaires. The first, "Modifiable Activity Questionnaire” (MAQ) [20], is used to measure the quantity of physical activity of the past year. The values are expressed as average (hours/week). The second, "Historical Leisure Activity Questionnaire” (HLAQ) [20], estimates the amount of regularly practiced activity throughout life. Data collected from the MAQ and HLAQ questionnaires regarding physical activity were presented in Table $\mathbf{1}$. Scores of the two questionnaires were converted to Met's [27], following the limit of questionnaires used in this study (i.e., the measure of Maximal oxygen consumption: VO2max). The Met ("Metabolic Equivalent") is equivalent to the corresponding metabolic oxygen consumption per unit body mass at rest [28].

\subsection{Experimental Design}

Before the beginning of the experimental protocol, subjects were familiarized with the procedures and tests involved in the study. During the experimental session, they performed two types of tasks (i.e., simple and complex). Tasks consisted of 72 trials of simple tests interrupted by a pause, followed by 72 trials of complex tests. Subjects perceived visual stimuli through the computer screen.

Concerning simple task stimulus (S1), parameters are specified by triplet alphabetic test (A, B, C... Y, Z) based on specific protocols and subjects' responses during those protocols. The font is adopted in "Times New Roman”. (S1) appears in black on a white screen (computer screen 15). Eye screen distance is approximately $46 \mathrm{~cm}$. At this distance, the character size is a vertical visual angle of $1.49^{\circ}$ and a horizontal visual angle of $1.04^{\circ}$.

The complex task was characterized by an increase of the complexity of the task by increasing the number of items to compare. The stimulus (S2) is a quadrupled alphabetic character, instead of a triplet. The time allocated

Table 1. Characteristics of the subjects (Mean \pm SD).

\begin{tabular}{cccc}
\hline & \multicolumn{2}{c}{ Young adults } & Older adults \\
\cline { 2 - 4 } & Sedentary & Active & Sedentary \\
Age (years) & $24.70 \pm 2.47$ & $23.75 \pm 2.80$ & $74.30 \pm 4.46$ \\
MAQ (h/week) & $1.59 \pm 1.36$ & $7.05 \pm 2.01$ & $1.23 \pm 0.85$ \\
HLAQ (h/week) & $3.59 \pm 2.45$ & $13.97 \pm 4.33$ & $3.10 \pm 2.93$ \\
MAQ MET's (h/week) & $8.36 \pm 5.45$ & $52.35 \pm 13.05$ & $5.47 \pm 3.89$ \\
HLAQ MET's (h/week) & $24.78 \pm 2.86$ & $103.94 \pm 23.25$ & $43.39 \pm 6.77$ \\
\hline
\end{tabular}


to the response remains unchanged. The task consisted of measuring the reaction time (RT) required by manual response. The RT was used as an index of individuals' motor performance. The test consisted of responding to a visual stimulus during each task. The subject was instructed to press the left/right button when the correct stimulus was perceived. There are two types of answers: the letter " $H$ " if the stimuli presented are identical and the letter " $V$ " if the stimuli presented are different. Each trial began with the appearance of a fixation point (an asterisk) in black for $500 \mathrm{~ms}$ from the centre of the screen. To avoid the anticipated responses, a preparatory period variable (blank screen) is introduced between the fixation point and the stimulus, during which no response is accepted. This preparatory period varies randomly between $100 \mathrm{~ms}$ and $250 \mathrm{~ms}$ in $50 \mathrm{~ms}$ block. The TR below 200 ms and above 2000 ms are considered "error" and are excluded from the analyzes of TR. Instructions and stimuli were presented with a Pentium 4 PC, with standard keyboards and E-prime software which recorded accuracy and response time. The stimulus disappears when a reply is given. Following the subject's response, feedback is displayed for $800 \mathrm{~ms}$.

\subsection{Statistical Analyses}

All statistical tests were processed using Statistica Software (StatSoft, Paris, France). Data are reported as mean \pm SD (standard deviation). Reaction time data were analyzed using a three-way ANOVA (2(Age) $\times 2$ (Complexity) $\times 2$ (Physical activity) $)$. When appropriate, significant differences between means were assessed using the LSD post hoc test. The level of statistical significance was set at $p<0.05$.

\section{Results}

The analysis of variance revealed significant age $\left(F_{(1116)}\right.$ $\left.=111.31 ; p<0.05 ; \eta^{2}=0.49\right)$, physical activity $\left(F_{(1116)}=\right.$ $\left.24.39 ; p<0.05 ; \eta^{2}=0.17\right)$, and complexity $\left(F_{(1116)}=\right.$ 324.15; $\left.p<0.05 ; \eta^{2}=0.74\right)$ effects. However, no significant age $\times$ complexity $\times$ physical activity interaction was observed for RT.

The age $\times$ physical activity interaction for RT to making-decision was significant $\left(F_{(1116)}=4.41 ; p<0.05 ; \eta^{2}=\right.$ $0.04)$. The post hoc analysis showed that older active subjects have lower RT than older sedentary subjects ( $p$ $<0.05$ ). However, no significant difference was observed in young adults. Moreover, young adults had signifycantly lower RT than older subjects $(p<0.05)$ (Figure 1).

The analysis of variance revealed also a significant age $\times$ complexity interaction for RT $\left(F_{(1116)}=37.75, p<0.05\right.$, $\left.\eta^{2}=0.25\right)$. The post hoc test showed a significant increase in the RT when task is complex compared to the simple task in all groups (Figure 2).

Also, the analysis of variance revealed a significant physical activity $\times$ complexity interaction $\left(F_{(1116)}=4.30\right.$, $\left.p<0.05, \eta^{2}=0.04\right)$. Active adults have better RT regardless the complexity of the task (i.e., simple or complex) (Figure 3).

\section{Discussion}

The aim of this study was to evaluate the impact of age, the practice of regular physical activity, and the task complexity on decision-making ability.

Our results showed that older active subjects have lower RT than older sedentary subjects. However, no significant difference was observed in young adults. Moreover, young adults had significantly lower RT than older subjects. Furthermore, we found a significant increase in the RT when task is complex compared to the simple task in all groups. In addition, active adults have

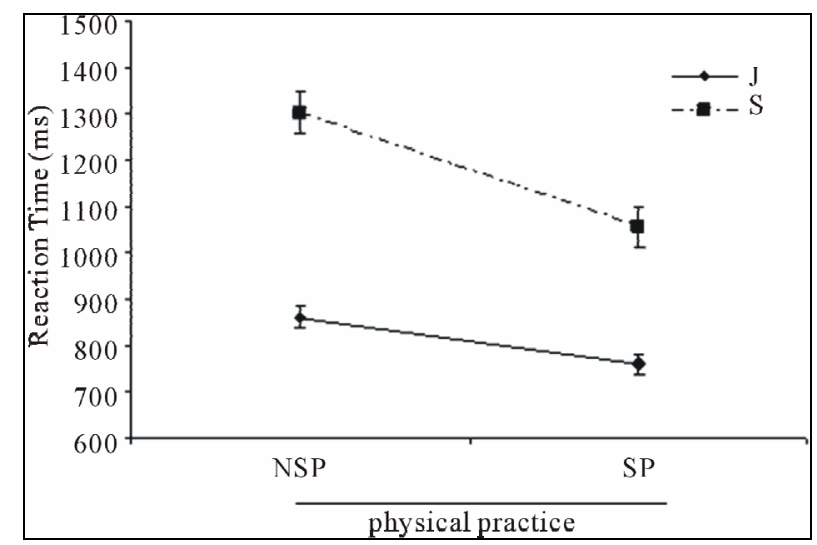

Figure 1. Mean $( \pm \mathrm{SD})$ reaction time (ms) for sedentary and active young adults, and sedentary and active older adults during decision-making.

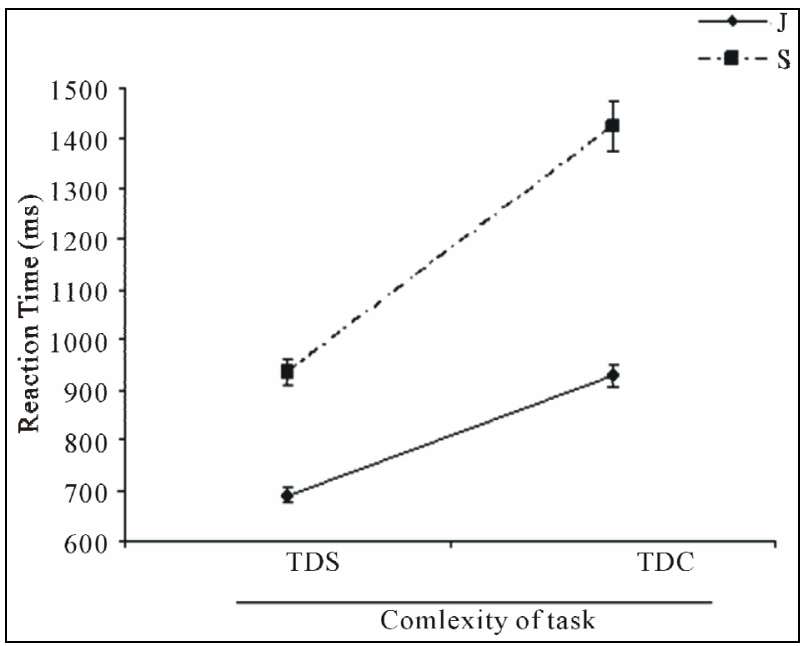

Figure 2. Mean $( \pm \mathrm{SD})$ reaction time $(\mathrm{ms})$ for sedentary and active young adults, and sedentary and active older adults during decision-making. 


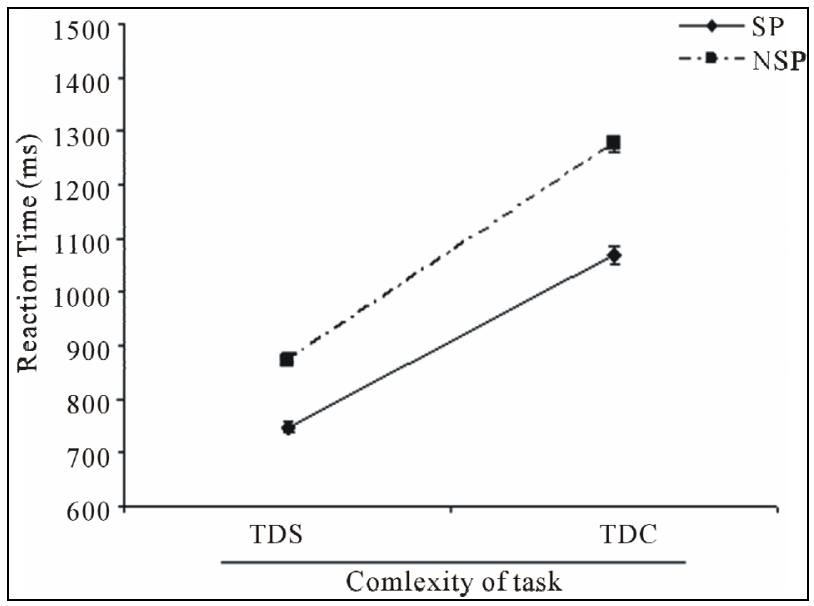

Figure 3. Mean $( \pm \mathrm{SD})$ reaction time (ms) for sedentary and active young adults, and sedentary and active older adults during decision-making.

better RT regardless the complexity of the task (i.e., simple or complex).

Our results showed that older adults took more time than young adults to respond to the stimulus during the decision-making task. Our results are consistent with several studies $[29,30]$ that showed alteration in cognitive ability due to aging. Among many cognitive skills, speed of information processing is considered to be especially prone to aging effects. Prior studies have shown a significant increase in RT between 20 and 60 year olds $[17,31]$, and this is in line with the results obtained in this study. Since many of these studies were performed with older healthy subjects without signs of mild cognitive impairment or dementia, the increase in RT might simply represent the effects of normal aging on basic cognitive function. In a number sequences task, Salthouse and Prill [32] found that young subjects took less time to complete the series. Moreover, the difference between the young and the elderly increased with difficulty of the series. Hence, we speculate that the increased mean RT could be the result of both decreased nerve transmission speed with age as well as the age-related decline in white matter integrity. Another possible explanation is that the reduction in time of practice of physical activity as a consequence of aging may affect the process of decision making. In agreement, it has been demonstrated that physical activity appears to be beneficial to aging since it improves processing capacities, executive control, and processing speed information as well as the decision making [33,34].

Furthermore, our results showed that active subjects have better RT compared to sedentary. This is consistent with the findings of Spirduso [35] who compared the performance of handball players (i.e., young and old) to inactive or sedentary subjects during simple and choice reaction time tasks. The decision time and production response were better in athletes than in sedentary. Similarly, active older adults had better cognitive performance compared to sedentary older adults. Our results are in line with those of Spirduso [36] and Clarkson-Smith and Hartley [17] that showed better performance in active older adults than sedentary (i.e., lower reaction time on simple and choice tasks). This beneficial effect of regular physical activity on the elderly goes against TOP model developed by Stones and Kozma [37], which predicts that active subjects, regardless of age, are better than their sedentary subjects on making decision. Also, the moderator model [38] suggests that physical activity plays an important role with aging. Consequently, physical activity would limit the impacts of natural aging on cognitive functioning of individuals.

Aging individual is characterized by a decrease in the efficiency and speed of cognitive processes. This decrease is even more important than the task at hand is complex [38]. Thus, the inter-individual differences are amplified by the increasing complexity of the decision task. The effect of task complexity also appears to be sensitive to these differences. These results are online with those of Salthouse et al. [34] that conducted a major research program studying the effects of aging on deductive reasoning. In this task, participants saw one to three premises and a question. They were asked if the answer was "yes" or "no". The number of premises was manipulated to determine whether the effect of aging was more important to more complex problems (i.e., more than premises behaviour). Indeed, performance on more complex problems is more affected by aging. The age $x$ complexity interaction showed no effect of aging, while for problems with two and especially three premises, the number of problems solved correctly decreases with age $[39,40]$.

Regarding the age $\times$ complexity $\times$ inactivity interaction, our results showed no interaction with the decision-making task. Unlike the study Abourezk and Tool [41], it seems that the complexity of the task may be an important factor in determining the relationship between physical activity and cognitive performance in elderly. Moreover, our results were not been able to reveal this larger effect of physical activity on complex tasks requiring expensive treatments sensible [42,43].

\section{Conclusion}

The reduction in decision making ability observed in this study in older adults is ascribed to aging-related changes in the underlying cognitive capacities. Also, physical activity improves the decision making ability in older subjects. Therefore, physical activity is beneficial for elderly. Furthermore, it would be pertinent to assess the impact of other potential moderating factors that could help maintaining better cognitive capacities. 


\section{Acknowledegments}

The authors wish to express their sincere gratitude to all the participants for their maximal effort and cooperation. This study was financially supported by the Ministry of Higher Teaching and Scientific Research, Tunisia.

\section{REFERENCES}

[1] S. Resnick, D. Pham, A. Kraut, A. Zonderman and C. Davatzikos, "Longitudinal Magnetic Resonance Imaging Studies of Older Adults: A Shrinking Brain,” Journal of Neuroscience, Vol. 23, No. 8, 2003, pp. 3295-3301.

[2] N. Raz, U. Lindenberger, K. Rodrigue, K. Kennedy, D. Head, A. Williamson, C. Dahle, D. Gerstorf and J. Acker, "Regional Brain Changes in Aging Healthy Adults: General Trends, Individual Differences and Modifiers," Cerebral Cortex, Vol. 15, No. 11, 2005, pp. 1676-1689. doi:10.1093/cercor/bhi044

[3] I. Driscoll, C. Davatzikos, Y. An, X. Wu, D. Shen, M. Kraut and S. Resnick, "Longitudinal Pattern of Regional Brain Volume Change Differentiates Normal Aging from MCI,” Neurology, Vol. 72, No. 22, 2009, pp. 1906-1913. doi:10.1212/WNL.0b013e3181a82634

[4] A. Fjell, K.Walhovd, C. Fennema-Notestine, L. Mcevoy, D. Hagler, D. Holland, J. Brewer and A. Dale, "One-Year Brain Atrophy Evident in Healthy Aging," Journal of Neuroscience, Vol. 29, No. 27, 2009, pp. 15223-15231. doi:10.1523/JNEUROSCI.0115-09.2009

[5] G. Bartzokis, J. Cummings, D. Sultzer, V. Henderson, K. Nuechterlein and J. Mintz, "White Matter Structural Integrity in Healthy Aging Adults and Patients with Alzheimer Disease: A Magnetic Resonance Imaging Study,” Archives of Neurology, Vol. 60, No. 3, 2003, pp. 393- 398. doi:10.1001/archneur.60.3.393

[6] F. Gunning-Dixon, A. Brickman, J. Cheng and G. Alexopoulos, "Aging of Cerebral White Matter: A Review of MRI Findings," International Journal of Geriatric Psychiatry, Vol. 24, No. 2, 2009, pp. 109-117. doi:10.1002/gps.2087

[7] C. Morse, "Does Variability Increase with Age? An Archival Study of Cognitive Measures," Psychology and Aging, Vol. 8, No. 2, 1993, pp. 156-164. doi:10.1037/0882-7974.8.2.156

[8] R. Wilson, L. Beckett, L. Barnes, J. Schneider, J. Bach, D. Evans and D. Bennett, "Individual Differences in Rates of Change in Cognitive Abilities of Older Persons," Psychology and Aging, Vol. 17, No. 2, 2002, pp. 179-193. doi:10.1037/0882-7974.17.2.179

[9] C. Van Petten, E. Plante, P. Davidson, T. Kuo, L. Bajuscak and E. Glisky, "Memory and Executive Function in Older Adults: Relationships with Temporal and Prefrontal Gray Matter Volumes and White Matter Hyperintensties,” Neuropsychologia, Vol. 42, No. 10, 2004, pp. 1313-1335. doi:10.1016/j.neuropsychologia.2004.02.009

[10] R. Verrillo, "Effects of Aging on the Suprathreshold Responses to Vibration,” Perception \& Psychophysics, Vol. 32, No. 1, 1982, pp 61-68. doi:10.3758/BF03204869
[11] G. Gescheider, S. Bolanowski, K. Hall, K. Hoffman and R. Verrillo, "The Effects of Aging on Information-Processing Channels in the Sense of Touch: I . Absolute Sensitivity," Somatosensory and Motor Research, Vol. 11, No. 4, 1994, pp. 345-357.

doi:10.3109/08990229409028879

[12] R. Verrillo, S. Bolanowski and G. Gescheider, "Effect of Aging on the Subjective Magnitude of Vibration," Somatosensory and Motor Research, Vol. 19, No. 3, 2002, pp. 238-244. doi:10.1080/0899022021000009161

[13] Y. Lin, S. Hsieh, C. Chao, Y. Chang and S. Hsieh, "Influence of Aging on Thermal and Vibratory Thresholds of Quantitative Sensory Testing," Journal of Peripheral Nervous System, Vol. 10, No. 3, 2005, pp. 269-281. doi:10.1111/j.1085-9489.2005.10305.x

[14] H. Dinse, N. Kleibel, T. Kalisch, P. Ragert, C. Wilimzig and M. Tegenthoff, "Tactile Coactivation Resets AgeRelated Decline of Human Tactile Discrimination," Annals of Neurology, Vol. 60, No. 1, 2006, pp. 88-94. doi:10.1002/ana.20862

[15] P. Greenwood, "Functional Plasticity in Cognitive Aging: Review and Hypothesis,” Neuropsychology, Vol. 21, No. 6, 2007, pp. 657-673. doi:10.1037/0894-4105.21.6.680

[16] P. Greenwood and R. Parasuraman, "Neuronal and Cognitive Plasticity: A Neurocognitive Framework for Ameliorating Cognitive Aging," Frontiers in Aging Neurosciene, Vol. 2, No. 150, 2010. doi:10.3389/fnagi.2010.00150

[17] P. Lemaire and L. Bherer, "Psychologie du Vieillissement. Une Approche Cognitive,” De Boeck Université, Bruxelles, 2005.

[18] D. Park and A. H. Gutchess, “Aging, Cognition and Culture: A Neuroscientific Perspective,” Neurosciences Biobehavioral Review, Vol. 26, No. 7, 2002, pp. 859-867.

[19] L. Clarkson-Smith and A. Hartley, "Relationships between Physical Exercise and Cognitive Abilities in Older Adults," Psychology and Aging, Vol. 4, No. 2, 1989, pp. 183-189. doi:10.1037/0882-7974.4.2.183

[20] K. Yaffe, D. Barnes, M. Nevitt, L. Lui and K. Covinsky, "A Prospective Study of Physical Activity and Cognitive Decline in Elderly Women-Women Who Walk," Archives of Internal Medicine, Vol. 161, No. 14, 2001, pp. 17031708. doi:10.1001/archinte.161.14.1703

[21] C. Emery and M. Gatz, "Psychological and Cognitive Effects of an Exercise Program for Community-Residing Older Adults,” The Gerontologist, Vol. 30, No. 2, 1990, pp. 184-188. doi:10.1093/geront/30.2.184

[22] L. Clarkson-Smith and A. Hartley, "Structural Equation Models of Relationships between Exercise and Cognitive Abilities," Psychology and Aging, Vol. 5, No. 3, 1990, pp. 437-446.

[23] D. Bunce, “The Locus of Age X Health-Related Physical Fitness Interactions in Serial Choice Responding as a function of Task Complexity, Central processing or Motor Function?” Experimental Aging Research, Vol. 27, No. 1, 2001, pp. 103-122.

[24] J. Etnier and D. Landers, "Motor Performance and Motor Learning as a Function of Age and Fitness," Research 
Quarterly for Exercise and Sport, Vol. 69, No. 2, 1998, pp. 136-146.

[25] J. L. Etnier, D. H. Romero and T. Traustadottir, “Acquisition and Retention of Motor Skills as a Function of Age and Aerobic Fitness," Journal of Aging and Physical Activity, Vol. 9, No. 4, 2001, pp. 425-437.

[26] W. Spirduso, H. MacRae, P. MacRae, J. Prewitt and L. Osborne, "Exercise Effects on Aged Motor Function," Annals of the New York Academy of Sciences, Vol. 515, No. 1, 1988, pp. 363-375.

[27] A. Kriska, W. Knowler, R. LaPorte, A. Drash, R. Wing, S. Blair, P. Bennett and L. Kuller, "Development of Questionnaire to Examine Relationship of Physical Activity and Diabetes in Pima Indians,” Diabetes Care, Vol. 13, No. 4, 1990, pp. 401-411. doi:10.2337/diacare.13.4.401

[28] B. Ainsworth, W. Haskell, A. Leon, D. Jacobs, H. Montoye, J. Sallis and R. Paffenbarger, "Compendium of Physical Activity: Classification of Energy Costs of Human Physical Activities," Medicine \& Science in Sports \& Exercise, Vol. 25, No. 1, 1993, pp. 71-80 doi:10.1249/00005768-199301000-00011

[29] E. Fox and T. Mathews, "Bases Physiologiques de l'Exercice Physique,” Vigot, Paris, 1984.

[30] C. Ketcham and G. Stelmach, "Age-Related Declines in Motor Control,” In: J. E. Birren and K. W. Schaie, Eds., Handbook of the Psychology of Aging, Academic Press, San Diego, 2001, pp. 313-348.

[31] J. Fozard, M. Vercryssen, S. Reynolds, P. Hancock and R. Quilter, "Age Differences and Changes in Reaction Time: The Baltimore Longitudinal Study of Aging,” Journal of Gerontology, Vol. 49, No. 4, 1994, pp.179-189. doi:10.1093/geronj/49.4.P179

[32] R. Ratcliff, A. Thapar and G. Mckoon, "The Effects of Aging on Reaction Time in a Signal Detection Task," Psychology and Aging, Vol. 16, No. 2, 2001, pp. 323-341. doi:10.1037/0882-7974.16.2.323

[33] S. Colcombe and A. Kramer, "Fitness Effects on the Cognitive Function of Older Adults: A Meta-Analytic Study," Psychological science, Vol. 14, No. 2, 2003, pp. 125-130. doi:10.1111/1467-9280.t01-1-01430

[34] T. Salthouse and K. Prill, "Inferences about Age Impairments in Inferential Reasoning," Psychology and Aging, Vol. 2, No. 1, 1987, pp. 43-51. doi:10.1037/0882-7974.2.1.43

[35] W. Spirduso, "Physical Dimensions of Aging," 2nd Edtion, Human Kinetics, Leeds, 2005.

[36] W. Spirduso, "Physical Fitness, Aging and Psychomotor Speed: A Review," Journal of Gerontology, Vol. 35, No. 6, 1980, pp. 850-865. doi:10.1093/geronj/35.6.850

[37] M. Stones and A. Kozma, "Physical Activity, Age and Cognitive/Motor Performance,” In: L. Howe and C. J. Brainerd, Eds., Cognitive Development in Adulthood, Springer Verlag, New York, 1988, pp. 271-321. doi:10.1007/978-1-4612-3852-2 9

[38] J. Cerella, L. Poon and D. Williams, “Age and the Complexity Hypothesis,” In: L. W. Poon, Ed., Aging in the 1980, American Psychological Association, Washington, DC, 1980, pp. 332-340. doi:10.1037/10050-024

[39] J. Fisk and C. Sharp, "Syllogistic Reasoning and Cognitive Ageing," Quarterly Journal of Experimental Psychology, Vol. 55, No. 4, 2002, pp. 1273-1293. doi:10.1080/02724980244000107

[40] A. Gilinsky and B. Judd, "Working Memory and Bias in Reasoning across the Life Span,” Psychology and Aging, Vol. 9, No. 3, 1994, pp. 356-371. doi:10.1037/0882-7974.9.3.356

[41] T. Abourezk and T. Toole, "Effect of Task Complexity on the Relationship between Physical Fitness and Reaction Time in Older Women," Journal of Aging and Physical Activity, Vol. 3, No. 3, 1995, pp. 251-260.

[42] A. Baylor and W. Spirduso, "Systematic Aerobic Exercise and Components of Reaction Time in Older Women," Journal of Gerontology: Psychological Sciences, Vol. 43, No. 5, 1988, pp. 121-126.

[43] T. Toole, S. Park and H. Al Ameer, "Years of Physical Activity Can Affect Simple and Complex Cognitive/Motor Speed in Older Adults,” General Electric, Fairfield, 1993. 